\title{
Instrumentos e o "saber-fazer" matemático no século $\mathrm{XVI}^{1}$
}

\author{
Instruments and "knowing by doing" of mathematics in the sixteenth century
}

Fumikazu Saito ${ }^{2}$

\section{RESUMO}

Este trabalho é dedicado aos instrumentos matemáticos do século XVI. Pautado em tendências historiográficas atualizadas, neste estudo examinamos o instrumento como artefato que incorpora conhecimentos do saber e do fazer matemáticos de uma época e suas implicações nas origens da ciência moderna. Este trabalho tem por base pesquisas e levantamentos pautados em fontes primárias. Especial atenção é dada ao báculo (baculum), um instrumento muito utilizado por agrimensores, navegadores e astrônomos. O báculo é analisado neste trabalho além de sua materialidade, considerando-se não só o instrumento, mas também os tratados que versam sobre ele. O estudo contextualizado de instrumentos matemáticos como o báculo revela importantes aspectos do processo da construção de conhecimento técnico e científico, conduzindo-nos a novas questões acerca da relação entre arte (techné) e ciência.

Palavras-chave: História da ciência. História da matemática. História da técnica. Instrumentos matemáticos. Báculo (baculum).

\begin{abstract}
This work is devoted to the sixteenth century mathematical instruments. Once based in the current trends in historiography, this study examine the instrument as an artifact which embodies knowledge of knowing by doing mathematics at a certain time, along with its implications in the early modern science. This work is based on primary-source researches and surveys. Special attention is given to the cross-staff (baculum), an instrument often used by surveyor, seamen and astronomers. The crossstaff is examined here beyond its materiality not only taking it into account but also considering treatises which deal with it. A contextualized study of mathematical instruments such as cross-staff reveals important aspects of the process of constructing technical and scientific knowledge leading us to new issues concerning the relationship between arts (techné) and science.
\end{abstract}

Keywords: History of science. History of mathematics. History of technology. Mathematical instruments. Cross-staff (baculum).

\footnotetext{
${ }^{1}$ Trabalho apresentado no dia 17 de outubro de 2013, no Grupo de Trabalho 10 - Ciência e Techné na História.

2 Pós-doutor em História da Ciência pela Pontifícia Universidade Católica de São Paulo (PUCSP); professor do Programa de Estudos Pós-Graduados em História da Ciência/CESIMA/PUCSP; e do Programa de Estudos Pós-Graduados em Educação Matemática/HEEMa/PUCSP; Doutorado em História da Ciência. E-mail: fsaito@pucsp.br
} 


\section{INTRODUÇÃO}

O uso de aparatos na investigação dos fenômenos naturais é muito antigo, porém, a sua importância foi acentuada no início do século XVII, quando "novos instrumentos" passaram a ser concebidos em virtude da demanda por novos métodos matemáticos e experimentais ${ }^{3}$ (WARNER, 1994, 1990; KUHN, 1989; BENNET, 1986; VAN HELDEN, 1983). Tais instrumentos começaram a ser utilizados para facilitar não só a resolução de problemas matemáticos, observacionais e experimentais, mas também para mapear a vasta e nova natureza produzida artificialmente (ALFONSO-GOLDFARB; BELTRAN, 2006, 2002; TURNER, 1998; HACKMANN, 2003, 1989; DAUMAS, 1972). Dentre esses instrumentos, encontramos aqueles denominados "matemáticos", isto é, instrumentos que foram concebidos para medir aquilo que Aristóteles (1952) denominava "quantidades" (distâncias e ângulos) (BENNET, 2003, 1998, 1991). Esses instrumentos, que já eram fabricados desde o século XIII (HACKMANN, 2003), passaram a ser confeccionados em grande quantidade a partir do século XVI em virtude, provavelmente, do reconhecimento de que a matemática era essencial não só para resolver problemas de ordem prática, mas também para investigar os diferentes aspectos da natureza (SAITO, 2012a, 2011; BENNETT, 1998).

Podemos dizer que a fabricação desses instrumentos revitalizou as práticas matemáticas a partir de meados do século XV, dando-lhes não só mais visibilidade, mas também reforçando a associação entre filósofos naturais e outros artesãos, principalmente, os praticantes de matemáticas ${ }^{4}$. Um dos fatores que levou os praticantes de matemáticas, artesãos e estudiosos da natureza, geralmente patrocinados por príncipes, comerciantes, banqueiros e outros, a investirem na produção desses instrumentos está relacionada ao próprio contexto de época. As transações comerciais, a pequena indústria em pleno desenvolvimento, as operações bancárias, as questões militares, o aumento dos valores das terras, entre outros aspectos, impulsionaram o desenvolvimento de novas ferramentas pra lidar com a nova ordem econômica e social (SAITO; DIAS, 2011). Foi nessas

\footnotetext{
${ }^{3}$ Os termos "instrumento" e "aparato" são utilizados como sinônimos para se referir a qualquer artefato em seu sentido mais lato e não necessariamente designam o instrumento científico como hoje é admitido. Vide: SAITO (2011); WARNER (1990).

${ }^{4}$ Sobre praticantes de matemáticas, vide: Mosley (2009); Higton (2001) e Taylor (1954).
} 
circunstâncias que floresceram muitas oficinas dedicadas à fabricação de instrumentos matemáticos em várias regiões da Europa, notadamente, Louvain, Nuremberg, Florença, Londres, entre outras (CONNER, 2005).

Esses instrumentos matemáticos são apreciados pelos historiadores da ciência de diferentes maneiras. A relação entre as diferentes artes e a ciência nas origens da ciência moderna tem sido o foco das principais investigações históricas. Outros estudos dedicados a diferentes possibilidades de relação entre ciência e técnica, articulando aspectos teóricos e práticos e suas relações que conduziriam a outras formas de interação no processo da formulação da ciência moderna também são comuns. Além disso, juntamente com outros, comumente conhecidos como "filosóficos", historiadores da ciência têm procurado discuti-los na crescente interação entre filosofia natural e matemáticas-mistas (GABBEY, 1997; WARNER, 1994).

Neste trabalho, entretanto, apresentamos o instrumento matemático atravessado por problemáticas diversas. Com base em estudos pautados em tendências historiográficas atualizadas, analisamos aqui o instrumento em seu processo de transmissão e apropriação de conhecimento (KUSUKAWA; MACLEAN, 2006), mais especificamente, como suporte que veicula conhecimentos do saber e do fazer matemáticos de uma época e suas implicações no desenvolvimento de novos conhecimentos nas origens da ciência moderna (SAITO, 2012b).

\section{INSTRUMENTO MATEMÁTICOS}

Instrumentos antigos são mais do que peças de antiquário. Para que possamos compreendê-los, é necessários inseri-los nos contextos em que foram gestados e flagrar no processo da construção do conhecimento seu real significado, sem atribuir-Ihes características notoriamente modernas. Análises e estudos em História da Ciência têm revelado que esses instrumentos não são meros objetos, mas artefatos que incorporam conhecimentos matemáticos e, portanto, não devem ser vistos como neutros, pois a sua construção e seu uso apontam para aspectos importantes do fazer matemático de uma época (SAITO; DIAS, 2011). 
Podemos dizer que os instrumentos passaram a ser vistos pelos historiadores da ciência como construtores de conhecimento e não apenas como objetos mediadores entre teoria e prática nos últimos trinta anos (TAUB, 2009; HANKINS, SILVERMAN, 1997; VAN HELDEN, HANKINS, 1994). Mais do que simples objetos, os instrumentos teriam, assim, importante papel no desenvolvimento de novos conhecimentos na medida em que exerceriam diferentes funções na articulação entre a teoria e a prática (GALISON, 1998). É nesse sentido que dizemos que os instrumentos matemáticos dos séculos XVI e XVII incorporam conhecimentos, tácitos e codificados, de sua época (SAITO, 2011). Cabe aqui observar, entretanto, que não se trata de conceber o instrumento como encarnação de conhecimento científico ${ }^{5}$, mas como um artefato que "incorpora" um conhecimento e, consequentemente, uma concepção de ciência (ou matemática) e de arte (techné). Isso porque tais artefatos comumente são resultados do rearranjo da manipulação de materiais e de ideias (GESSNER, 2010; HARKNESS, 2007; HINDLE, 1981).

Isso significa que, num instrumento, não seria bem a ciência (ou matemática) que determinaria como os seus componentes deveriam ser arranjados e organizados, embora a sua função, enquanto instrumento, esteja vinculada aos propósitos para os quais foi concebido e enquadrada nos moldes de uma teoria científica ou matemática em particular. Para que possamos examinar um instrumento matemático de forma contextualizada, é necessário que consideremos que os instrumentos e os tratados que versam sobre eles descrevem e transmitem "materialmente" informações a respeito de seu passado. Em outros termos, tais instrumentos devem ser estudados não só em relação aos que eles são (em sua materialidade), mas também em relação a como eles foram utilizados, fabricados e disseminados.

Convém observar que a construção e o uso de um instrumento mobilizam vários tipos de conhecimento relativo não só a sua materialidade, mas também às diferentes formas de manipulação e adequação físicas para ser utilizado na prática. Assim complexas redes de relações entre ciência, matemática, arte e sociedade encontram-se embebidas nos projetos, na confecção e nos dados sobre as formas e

\footnotetext{
${ }^{5}$ Sobre o instrumento como "encarnação" de teorias, vide: Koyré (1991, p. 193) e Bachelard (1977, p. 129).
} 
propósitos de construção e utilização desses instrumentos. Compreendidos além de sua materialidade, os instrumentos matemáticos podem, dessa maneira, revelar conhecimentos na articulação entre o saber e o fazer e, assim, a produção de conhecimento de uma época. Conhecimento este que pode receber diferentes interpretações e, consequentemente, significados. Como abordaremos a seguir, o báculo (baculum) é um dos muitos instrumentos matemáticos que revelam aspectos importantes do saber e do fazer matemático, que encontram-se sintetizados no instrumento, do século XVI.

\section{O CASO DO BÁCULO}

Os instrumentos nos revelam que a associação entre estudiosos da natureza e da matemática com artesãos no desenvolvimento do conhecimento científico e matemático não esteve desvinculado de outros segmentos teóricos e operacionais. Podemos dizer que os praticantes de matemática, em geral, tinham uma orientação mais empirista, no sentido de que as técnicas matemáticas por eles desenvolvidas eram aplicadas ao mundo real. Muitos deles se denominavam "professores" de matemática, no sentido daquele que professava a arte matemática. A maioria deles, entretanto, não possuía formação universitária e estava associada a uma corporação de ofício, ou trabalhava em uma oficina que fabricava instrumentos. Desse modo, era muito comum que esse "profissional" desenvolvesse seu próprio instrumento e comunicasse a respeito de sua construção e uso apenas àqueles que procurassem a sua instrução em sua oficina ou numa escola de ábaco $(\mathrm{ClOCCl}$, 2009: 59-71; BIAGIOLI, 1989; VAN EGMOND, 1980). É nesse contexto, que vemos surgir novos tratados sobre antigos instrumentos matemáticos, tal como o báculo, utilizados para medir distâncias.

O báculo é um instrumento muito antigo. Contudo, sua primeira descrição sistemática remonta ao século XIV e é atribuída a Levi ben Gerson (1288-1344) (SIMONSON, 2013). Embora fosse um instrumento muito utilizado em astronomia, no século XVI vemos sua aplicação na agrimensura e, em alguns casos, na navegação. Isso é compreensível se considerarmos que esse dois campos de saber adquiram importância naquela época em virtude da descoberta de novas terras. 
Além disso, no século XVI, o sistema de cultivo da terra, a posse de novas terras e o aumento significativo de seu valor, conduziu muitos proprietários a buscar novas regras para determinar a quantidade de terra, seus limites e localização, reorganizando, dessa maneira, o espaço (FISCHER, 1996; RICHESON, 1966). Assim, os primeiros tratados de instrumentos utilizados para medir distâncias (comprimento, largura e profundidade) geralmente traziam instruções para os administradores e superintendentes de terras (RICHENSON, 1966). Mas, na medida em que avançamos do século XVI em direção ao XVIII, o número de tratados que buscavam instruir sobre técnicas de medida e de mapeamento de terras aumentara significativamente. Esses tratados geralmente apresentavam diferentes técnicas de medidas para diferentes situações. E, para cada situação, encontramos instruções para construção e uso de um instrumento específico. Além disso, alguns desses tratados apresentavam a validação matemática do instrumento, discorrendo sobre sua demonstração geométrica fundamentada nos axiomas, postulados e teoremas de Elementos de Euclides; ao passo que outros, apenas procuravam descrevê-lo, apresentando seus possíveis usos (SAITO, 2012b).

No caso do báculo, ele era utilizado ao lado de outros instrumentos matemáticos, tais como o quadrante num quarto de círculo, ou um quadrante geométrico, para medir distâncias (altura ou largura) inalcançáveis (BARTOLI, 1564; DIGGES, 1556). Cosimo Bartoli (1503-1572), em Del modo di misurare (1564), por exemplo, refere-se ao báculo como um instrumento que permite medir uma linha da qual não é possível se aproximar (BARTOLI, 1564, p. 20r).

O báculo é um instrumento interessante não só por sua configuração material, mas também pelos diferentes conceitos geométricos que mobiliza e pelas relações que estabelece entre diferentes formas de conhecimento. Além disso, comparado a outros instrumentos matemáticos, podemos dizer que sua construção é relativamente simples, embora o seu manuseio na realização de uma medida seja bastante complexo.

Este instrumento é composto basicamente de duas hastes (figura 1) em que as marcações na haste $A B$ correspondem à medida da haste $C D$, denominada transversal. Assim, a construção do instrumento requer apenas a habilidade de um carpinteiro que saiba cortar a madeira, fazer os encaixes, e imprimir as marcas no instrumento. 


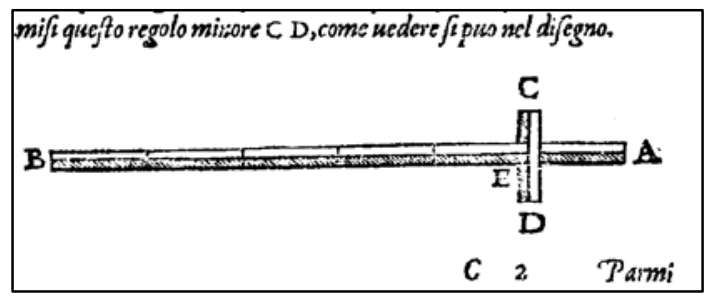

Figura 1- Báculo

Fonte: BARTOLI (1564, p. 10 r)

Contudo, a leitura das instruções para se proceder à medida nos revela que além de conhecimentos matemáticos, é necessário destreza ao manusear o instrumento. $\mathrm{O}$ que à primeira vista parece ser simples, pois basta apenas seguir as instruções dadas no tratado, revela-se bastante difícil de ser executada, visto que no processo de medição, a posição do observador no espaço, a posição do olho em relação ao instrumento e a posição do instrumento no espaço configuram três ações não triviais.

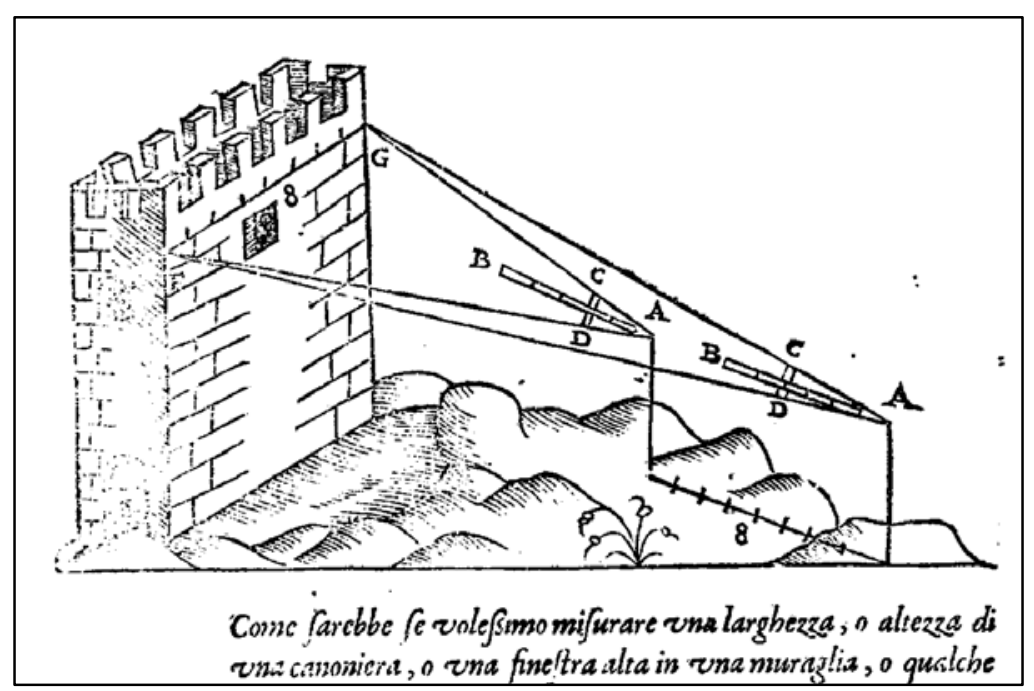

Figura 2 - Medindo a largura de um muro

Fonte: BARTOLI (1564, p. 11v)

Por exemplo, segundo Bartoli, para medir a largura de um muro FG (figura 2), basta que realizemos duas medidas em duas diferentes posições. Com o olho centrado em $A$, e a haste $C D$ localizada na primeira marca do instrumento na haste maior $A B$, realizamos a primeira medida, que consiste em encaixar a largura FG na 
largura $C D$ do instrumento. Em seguida, marcando-se a posição em que esta medida foi feita, devemos deslizar a haste $C D$ para uma nova posição no instrumento (haste $A B$ ) e, afastando-se do lugar em que a primeira medida foi realizada, devemos encontrar o lugar em que a largura $C D$ se encaixe novamente na largura FG. Marcamos assim este segundo lugar, e a medida da largura do muro corresponderá à distância entre o lugar em que foi realizada a primeira medida e a segunda, conforme figura 2 (BARTOLI, 11r).

O procedimento de medida em tese não é difícil de ser entendido, entretanto, é muito difícil de ser executado. Além do conhecimento matemático que está incorporado no instrumento, ou seja, as propriedades e as relações métricas nos triângulos isósceles, é necessário também treino no manuseio do instrumento. Além disso, ao afastar-se para realizar a segunda medida, é importante, por exemplo, que os dois triângulos AFG (formados na primeira e na segunda medida) estejam contidos num mesmo plano. $\mathrm{O}$ que significa que seria necessário apoiar o instrumento num tripé ou "mesa" para que a altura de A ao chão permanecesse a mesma. Essas e outras instruções, entretanto, não são explicitadas no tratado. Além disso, se considerarmos que o báculo era utilizado na navegação, é necessário também considerarmos a agitação do mar quando de seu uso. Tendemos a imaginar que esses instrumentos eram facilmente manipulados e esquecemos muitas vezes das circunstâncias nas quais eram utilizadas. Apontar o báculo para o céus $\mathrm{e}$ executar uma medida em alto-mar não era tarefa simples, mesmo dispondo de tripés e hastes para sustentar o instrumento. Era necessário, assim, a perícia do navegador que conhecia os procedimentos para executar a medida.

Isso significa que o corpo de quem mede fazia parte do processo de mensuração. Diferentemente dos modernos instrumentos, que geralmente realizam a medida com a mínima interferência de quem os manuseia (como uma régua, por exemplo), o báculo requer não só destreza, mas também conhecimentos matemáticos relativos à medida. Nesse particular, deve-se ressaltar que o princípio de medida no báculo é linear, ou seja, emprega escalas lineares de medida. O báculo dispensa, assim, o uso de ângulos para medir e, portanto, de tabelas 
trigonométricas que estavam em processo de reelaboração desde o século $\mathrm{XV} .{ }^{6} \mathrm{Em}$ termos de óptica, diríamos que o báculo se aproximava das concepções geométricas relacionadas à perspectiva linear, visto que não considerava a abertura visual (o ângulo formado em A) para realizar as medidas. A distância dos objetos na perspectiva linear não era calculada a partir do ângulo de visão, mas da distância em que se encontravam tais objetos.

\section{CONSIDERAÇÕES FINAIS}

O estudo de instrumentos, tal como o báculo, revela que ao abordar instrumentos e outros aparatos antigos é necessário considerá-los mais do que mera ferramenta. É necessário que as análises contextualizem o instrumento numa rede de relações, visto que é somente por meio de uma ampliação de foco, em que incidem análises que vão além da materialidade, que conseguimos captar a real expressão do conhecimento implicado nesses artefatos. Como muitos outros instrumentos desse período, o báculo que é essencialmente um instrumento matemático, não surgiu de uma necessidade matemática, mas foi resultado da prática matemática de uma época. Enquanto instrumento é produto da arte e da prática matemática que, num período posterior, adquiriria gradativamente importância matemática.

\section{REFERÊNCIAS}

ALFONSO-GOLDFARB, A. M.; BELTRAN, M. H. R. (Orgs.). O laboratório, a oficina e o ateliê: a arte de fazer o artificial. São Paulo: Educ/FAPESP/INEP, 2002.

O saber fazer e seus muitos saberes: experimentos, experiências e experimentações. São Paulo: Educ/Ed. Livraria da Física, FAPESP, 2006.

\footnotetext{
${ }^{6} \mathrm{O}$ uso de relações trigonométricas, entretanto, se tornaria comum ao longo do século XVI, quando novos instrumentos passariam a incorporar uma escala angular. Vide por exemplo: FINEO (1556) e CHATSFEILD (1650).
} 
ARISTÓTELES. Categories (Categoriae). In: Aristotle. The Works of Aristotle. Trad. E. M. Edghill. Chicago/London: Encyclopaedia Britannica, 1952, v. 2, p. 5-20 (Col. Great Books of the Western World, vol. 8).

BACHELARD, G. O racionalismo aplicado. Rio de Janeiro: Zahar, 1977.

BARTOLI, C. Cosimo Bartoli Gentil'huomo, et accademico Fiorentino, Del modo di misurare le distantie, le superficie, i corpi, le piante, le province, le prospettive (...). Venetia: Francesco Franceschi Sanese, 1564.

BENNETT, J. A. The Mechanics' Philosophy and the Mechanical Philosophy. History of Science, v. 24, p. 1-28, 1986.

. The challenge of practical mathematics. In: PUMFREY, S.; ROSSI, P. L.; SLAWINSKI, M. (Orgs.). Science, Culture and Popular Belief in Renaissance Europe. Manchester/New York: Manchester University Press, 1991. p. 176-190. $\overline{222,1998 .}$

Practical Geometry and Operative Knowledge. Configurations, v. 6, p. 195. Knowing and doing in the sixteenth century: what were instruments for?

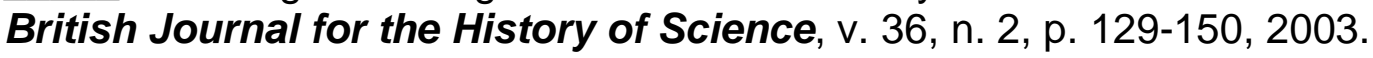

CHATSFEILD, J. The trigonall sector: The description and use thereof: Being an instrument most aptly serving for the resolution of all Rightlined Triangles with great faculty and delight (...). London: Robert Leybourn, 1650.

CONNER, C. D. A People's History of Science: Miners, Midwives, and "Low Mechanicks". New York: Nation Books, 2005.

DAUMAS, M. Scientific Instruments of the 17th \& 18th Centuries and their Makers. London: Portman Books, 1972.

DIGGES, L. A boke named Tectonicon. Briefelye shewynge the exacte, and speady reckenynge all manner lande, squared tymber, stone, steaples, pyllers, globes, etc.... London: Iohn Daye for Thomas Gemini, 1556.

FINEO, O. La composition et usage du Quarre Geométrique (...). Paris: Gilles Gourbin, 1556.

FISCHER, D. H. The Great Wave: Price, Revolution and the Rhythm of History. New York/Oxford: Oxford University Press, 1996. p. 65-91.

GABBEY, A. Between ars and philosophia naturalis: reflections on the historiography of early moderns mechanics. In: FIELD, J. V.; JAMES, F. A. J. L. (Orgs.).

Renaissance \& Revolution: Humanists, Scholars \& Natural Philosophers in Early Modern Europe. Cambridge: Cambridge University Press, 1997. p. 133-145. 
GALISON, P. L. History, Philosophy, and the Central Metaphor. Science in Context, v. 2, n.1, p. 197-212, 1988.

GESSNER, S. Savoir manier les instruments: la géometrie dans les écrits italiens d'architecture (1545-1570). Revue d'Histoire des Mathématiques, v. 16, n.1, p. 87147, 2010.

HACKMANN, W. D. Scientific Instruments: Models of Brass and Aids to Discovery. In: GOODING, D.; PINCH, T.; SCHAFFER, S. (Orgs.). The Uses of Experiment: Studies in the Natural Sciences. Cambridge/New York: Cambridge University Press, 1989. p. 39-43.

. Natural Philosophy and the Craft Techniques of Experimentation. Bulletin of the Scientific Instrument Society, v. 78, p. 35-37, 2003.

HANKINS, T. L.; SILVERMAN, R. J. Instruments and the Imagination. Princeton: Princeton University Press, 1997.

HARKNESS, D. E. The Jewel House: Elizabethan London and the Scientific Revolution. New Haven/London: Yale University Presss, 2007. p. 97-141.

HIGTON, H. Does using an instrument make you mathematical? Mathematical practitioners of the $17^{\text {th }}$ century. Endeavour, v. 25, n. 1, p. 18-22, 2001.

HINDLE, B. Emulation and Invention. New York: New York University Press, 1981.

KOYRÉ, A. Estudos de história do pensamento científico. $2^{\mathrm{a}}$ ed. Rio de Janeiro: Forense Universitária, 1991.

KUHN, T. S. Tradição matemática versus tradição experimental no desenvolvimento da ciência física. In: KUHN, T. S. A tensão essencial. Lisboa: Edições 70, 1989. p. 63-100.

KUSUKAWA, S.; MACLEAN, I. (Eds.). Transmitting Knowledge: Words, Images, and Instruments in Early Modern Europe. Oxford/New York: Oxford University Press, 2006.

MOSLEY, A. Early Modern Cosmography: Fine's Sphaera Mundi in Content and Context. In: MARR, A. (Org.). The Worlds of Oronce Fine: Mathematics, Instruments and Print in Renaissance France. Donington: Shaun Tyas, 2009. p. 114136.

RICHESON, A. W. English Land Measuring to 1800: Instruments and Practices. Cambridge/London: The Society for the History of Technology/The MIT Press, 1966. p. 189.

SAITO, F. O telescópio na magia natural de Giambattista della Porta. São Paulo: EDUC/Ed. Livraria da Física/FAPESP, 2011. 
. Possíveis fontes para a História da Matemática: Explorando os tratados que versam sobre construção e uso de instrumentos "matemáticos" do século XVI. In: SILVA, M. R. B. da; HADDAD, T. A. S. (Orgs.). 13 Seminário Nacional de História da Ciência e da Tecnologia - FFLCH USP - 03 a 06 de setembro de 2012. Anais. São Paulo: EACH/USP, 2012a. p. 1099-1110.

History of Mathematics and History of Science: Some remarks concerning contextual framework. Educação Matemática Pesquisa, v. 14, n. 3, p. 363-385, 2012b.

SAITO, F.; DIAS, M. S. Articulação de entes matemáticos na construção e utilização de instrumento de medida do século XVI. Natal: Sociedade Brasileira de História da Matemática, 2011.

SIMONSON, S. The Mathematics of Levi ben Gershon, the Ralbag. Disponível em: <http://u.cs.biu.ac.il/ tsaban/Pdf/MathofLevi.pdf>. Acesso em: 6 jul. 2013.

TAUB, L. On Scientific Instruments. Studies in History and Philosophy of Science, v. 40, p. 337-343, 2009.

TAYLOR, E. G. R. The Mathematical Practitioners of Tudor \& Stuart England. Cambridge: Institute of Navigation/Cambridge University Press, 1954.

TURNER, G. L'E. Scientific Instruments 1500-1900. An Introduction. Berkeley/Los Angeles/London: University of California Press, 1998.

VAN EGMOND, W. Practical Mathematics in the Italian Renaissance: A Catalog of Italian Abbacus Manuscripts and Printed Books to 1600. Annali dell'Istituto e Museo di Storia della Scienza, fasc. 1, p. 3-33, 1980.

VAN HELDEN, A. The Birth of the Modern Scientific Instrument, 1550-1770. In: BURKE, J. G. (Org.). The Uses of Science in the Age of Newton. Berkeley/Los Angeles/London: University of California Press, 1983. p. 49-84.

VAN HELDEN, A.; HANKINS, T. L. Hankins. Introduction: Instruments in the History of Science. Osiris, v. 9, p. 1-6, 1994.

WARNER, D. J. What is a scientific instrument, when did it become one, and why? British Journal for the History of Science, v. 23, pp. 83-93, 1990.

. Terresterial Magnetism: For the Glory of God and the Benefit of Mankind.

Osiris, v. 9, p. 67-84, 1994. 